This is a pre-print of an article published in Perspectives on Behavior Science. The final authenticated version is available online at: https://doi.org/10.1007/s40614-022-00332-3

\title{
Some Characteristics and Arguments in Favor of a Science of Machine Behavior Analysis
}

\author{
Marc J. Lanovaz ${ }^{1,2}$ \\ ${ }^{1}$ École de psychoéducation, Université de Montréal \\ ${ }^{2}$ Centre de recherche de l'Institut universitaire en santé mentale de Montréal
}

\begin{abstract}
Author Note
We thank Antonia Giannakakos-Ferman and John T. Rapp for their comments on an earlier draft of this manuscript

Correspondence concerning this article should be addressed to Marc J. Lanovaz, École de psychoéducation, Université de Montréal, C.P. 6128, succursale Centre-Ville, Montreal, QC, Canada, H3C 3J7. Email: marc.lanovaz@umontreal.ca, Phone: 1 514-343-6111 \#81774
\end{abstract}




\begin{abstract}
Currently, researchers and practitioners recognize four domains of behavior analysis: radical behaviorism, the experimental analysis of behavior, applied behavior analysis, and the practice of behavior analysis. Given the omnipresence of technology in every sphere of our lives, the purpose of this conceptual paper is to describe and argue in favor of a fifth domain: machine behavior analysis. Machine behavior analysis is a science that examines how machines interact with and produce relevant changes in their external environment by relying on replicability, behavioral terminology, and the philosophical assumptions of behavior analysis (e.g., selectionism, determinism, parsimony) to study their artificial behavior. Arguments in favor of a science of machine behavior include the omnipresence and impact of machines on human behavior, the inability of engineering alone to explain and control machine behavior, and the need to organize a community of non-mentalistic scientists around a common issue. Regardless of whether behavior analysts agree or disagree with our position, we argue that we need to debate the topic. As such, the current article aims to encourage and contribute to this debate.
\end{abstract} Keywords: artificial intelligence, behavior analysis, machine learning 


\section{Some Characteristics and Arguments in Favor of a Science of Machine Behavior Analysis}

Since Skinner initially proposed a science of behavior in the 1930s, the world in which we live has evolved tremendously. One of the most notable changes is the pervasive presence of technology in our homes and our workplaces. To put things in perspective, researchers only built the first automatic digital computers in the 1940s (Watson \& Calhoun, 1960). These computers needed large rooms and could only conduct basic calculations to solve scientific problems. Skinner experimented with machines (albeit nondigital) such as the air crib, the teaching machine, and the operant conditioning chamber (i.e., often referred to as a Skinner box) during his lifetime (Skinner, 1958, 1961). He even discoursed on whether man and machines were really that different (Skinner, 1969). However, this early technology does not compare to the diversity of functions that contemporary computers can carry out. Nowadays, smartphones can not only fit in our pockets, but they are also significantly more powerful than early computers. As an example of the omnipresence of technology in our lives, $84 \%$ of Americans with a smartphone report consulting it within 15 min of getting up in the morning (Levitas, 2013).

In the past decades, the development and the application of new algorithms (i.e., sets of computer instructions that solve a problem) as well as progress in computing power have allowed machines to reach a point wherein electrical and computer engineers are oftentimes unable to predict how machines will "respond" given an input (Rudin, 2019; Sendak et al., 2021; Watson et al., 2019). In an example popularized by the media, Microsoft developed a Twitter chatbot, named Tay, which was supposed to learn to hold conversations online. After only $24 \mathrm{hr}$, the development team had to step in because Tay had learned "to tweet like a Nazi sympathizer, racist and supporter of genocide, amongst other things" (Wakefield, 2016). Based on their knowledge of machine learning algorithms and coding, the engineers could not predict Tay's 
behavior once left to fend on its own "in the wild". In this example, studying the "behavior" of the machine (i.e., a chatbot) when presented with different inputs could have prevented this issue. This article aims to argue that we need a science of machine behavior analysis to address this issue and many others that stem from rapid technological developments.

Currently, behavior analysts typically recognize four domains of behavior analysis: radical behaviorism, the experimental analysis of behavior, applied behavior analysis, and the practice of behavior analysis (Cooper et al., 2020; Moore, 2008). The focus of these domains is humans and non-human living organisms. Behavior analysis does not have a domain that focuses on the responding of machines to their external environment. The main thesis of this conceptual article is that behavior analysts need to formalize a fifth domain to address this issue: machine behavior analysis.

\section{Some Basic Characteristics}

Before making an appeal for the formalization of a science of machine behavior analysis, the first step is to establish potential boundaries for this science. In the current section, we describe the six basic characteristics of a science of machine behavior analysis. These characteristics should not be perceived as complete, nor exclusive, but rather as a starting point to better define the science.

\section{Machines as its Subject Matter}

As indicated in the introduction, the main distinction of machine behavior analysis is its emphasis on machines. Instead of focusing its efforts on the behavior of humans or other living organisms, the science focuses on machines as its own subject matter. The logical question that follows is: What do we mean by "machine"? To produce meaningful analyses for behavioral scientists, machines should meet three basic criteria: (a) they should produce an observable 
change in the environment following the presentation (or absence) of certain external events or stimuli, (b) they should not occur naturally, and (c) they should consider previously stored data when producing a change in the environment.

The first criterion is similar to what we would expect of any behaving organism. That is, the behavior analyst should have something to measure (i.e., a change in the environment). Without this attribute, there would be nothing for the behavior analyst to study. On its own, the first attribute is insufficient as a rock would meet this definition (i.e., when I push a rock on the edge of hill, it will start rolling down if I apply sufficient force). The second criterion eliminates the prior issue by requiring that the machine not occur naturally (i.e., a definition by exclusion). We preferred the expression "not occur naturally" to the expression "produced by humans" because machines may eventually build other machines and humans can produce other humans through reproduction. By definition, anything produced through cosmic evolution or natural selection would not be considered a machine. Once again, this definition remains incomplete as simple machines such as levers and pulleys would meet both these criteria. What sets machines meaningful to behavior analysts apart is the third criteria. Machines should consider data stored prior to exerting a change on the environment. This stored data can be provided to the machine by the engineer prior to its release, or the machine can record it automatically through its interaction with the environment. Based on these criteria, machines can encompass a broad array of apparatuses that humans interact with on a daily basis.

At this point, we should specify that machines involve two main components: hardware and software. Hardware is the physical apparatus that runs algorithms such as computers, smartphones, cars, smart speakers, and servers. Software (sometimes referred to as firmware in certain devices) includes the instructions, or algorithms, that tell the hardware what to do. The 
two components, hardware and software, interact together and dictate what a machine can and cannot do. For example, the hardware controls what type of input a machine may receive from its external environment whereas the software uses this data to transform the environment within the limits set by the hardware. Hence, two machines composed of different hardware, but with the same software, may still produce the same behavior. A calculator, a smartphone, and a desktop computer can all multiply numbers efficiently and produce an output of the response on a screen (functionally equivalent). In the same vein, the same hardware may run two different software (e.g., a computer may run a calculator and a music player). In the latter case, we would treat each as a different machine as their functions differ.

Figure 1 presents how the external environment, hardware and software may interact for a given machine. The first step involves the external environment providing an input to the machine, which is captured by the hardware. This input may be any environmental change that a machine perceives with its electronic sensors (part of the hardware). Examples include tactile (e.g., keyboard), visual (e.g., camera), auditory (e.g., a microphone), spatial orientation (e.g., gyroscope) and thermal (e.g., heat sensor) inputs. Next, the software uses the data collected by the hardware to determine the output. During this step, algorithms will transform part, or all, of the input data to produce a signal as output. Based on this signal, the hardware will typically produce a human-readable output. Similar to inputs, outputs may include tactile (e.g., a robot touching a surface or a person), visual (e.g., an image on a screen), auditory (e.g., a voice from a smart speaker), spatial orientation (e.g., a correction in position) and thermal (e.g., a heater) stimuli. The input and output do not need to measure and produce the same change in the environment. For example, a robot may take in an auditory input to produce a tactile output. In 
sum, machines are versatile objects that can use various types of input stimuli to produce different types of changes in their environment.

\section{Centered on Machine Behavior}

Now that we have a definition of a machine, we can move on to the second characteristic of a science of machine behavior analysis, centered on machine behavior. Whereas experimental and applied behavior analysis deal with the (natural) behavior of living organisms as dependent variables, machine behavior analysis should focus on machine behavior. We use the expression machine behavior as behavior on its own is already well-defined. That is, the term "behavior" implicitly applies to living organisms whereas "machine behavior" can be reserved for machines. A simple definition of machine behavior could be any observable change in the environment produced by a machine.

When conducting machine behavior analysis, scientists should focus on the behavior of the machine rather than on the behavior of the living organism. Human behavior still plays a role in a science of machine behavior analysis as it may serve as the independent variable. For example, how does a machine respond to the changes in the environment produced by the human experimenter? In this case, we manipulate the human behavior and examine its effects on the machine behavior. This approach is not unlike the other domains of behavior analysis: the main change is that the locus of our analysis is now the machine. We can also think of situations where both the behavior of the human and the behavior of the machine are studied simultaneously. This type of transversal study would involve both applied and machine behavior analysis to identify relations between human and machine behavior.

\section{Relevant}


From a behavior analytic perspective, not all machine behavior is relevant to study. The problem is that a machine may produce observable changes (machine behavior) that have limited relevance for behavioral scientists. For example, machine learning may project sets of numbers used by the algorithms on a computer screen. Similarly, interacting with a machine may produce observable changes in electric current. Both these machine behaviors can be observed by the behavior analyst, but we would argue that neither has a high relevance to them. In the same manner as the brain is the topic of physiological research, the internal state of the machine should remain the purview of engineers. To be relevant to a behavioral scientist, a machine behavior should be an environmental change designed to function as a specific antecedent or a consequence for its user; this user could be a human, a living organism or even another machine. For example, electric current and equations (even though observable) are not designed to function as antecedents and consequences for a layperson using Facebook. As such, the machine behavior could be labeled as irrelevant. Instead, relevant machine behavior may involve Facebook showing you a specific ad on your smartphone, recommending a new friend on a computer, or ordering the posts of your friends on a tablet.

A second dimension of relevance involves the predictability of the machine behavior. When we press on the letter " $\mathrm{k}$ " on the keyboard of a computer, the letter " $\mathrm{k}$ " always appears on our screen. Although the appearance of " $\mathrm{k}$ " on our screen is a machine behavior produced following some external event, its predictability makes its relevance very limited for behavioral scientists. If an algorithm produces responses with a known distribution (e.g., a random number generator returns values from a normal distribution), we will know what to expect if we repeat the process for a large number of trials. Put differently, a machine behavior is only relevant to study if we do not know a priori what specific patterns of responding should be observed. To go 
back to the Microsoft experiment, an example of relevant machine behavior to study is how Tay, the chatbot, changes the content of its tweets based on different human behavior. The programmers themselves where unaware how the external environment (i.e., the tweets of others) would artificially shape its tweeting behavior. A behavior analytic approach would have been well suited to study this type of issue.

\section{Replicable}

To ensure progress, any science should be replicable and machine behavior analysis makes no exception. Consistent with the dimensions of applied behavior analysis (Baer et al., 1968), a replicable science should make use of technological descriptions. Researchers must describe their procedures in sufficient details so that someone with training in the science could replicate their methodology. Another dimension of replicability involves using research methodologies that can produce reproducible results. The science should strive to show prediction and control over behavior. Most machines that engage in relevant behavior probably have their own idiosyncratic responding. Thus, we suspect that single-case methodology can play a central role in the development of the science. Single-case designs may not only facilitate within- and between-subject replications, but they may also control for idiosyncratic responding across machine subjects. That said, the science should not limit itself to one specific methodology. For example, researchers may use a reversal design to examine the effects of different navigating behaviors on the product recommendations made by Amazon.com, but a randomized controlled trial may be better suited to study how multiple different social robots respond to two specific interactions. The research question and purpose should select the methodology that will allow for the most convincing demonstration of prediction or control. 
Regardless of methodology, research designs used in machine behavior analysis should have the potential to produce reproducible results.

One concern regarding replicability is that each machine is programmed in a unique manner. Thus, replicating the same results across different machines may be a challenge. Behavior analysts are already aware of this issue as each species, as well as each individual within a species, is unique. For individuals within species, variations in responding may be explained by variations in the initial conditions. These initial conditions include organismspecific genes and prior contact of the individual organism with the environment over which the experimenter has no control. These variations are even larger across species because different species have distinct genes. Therefore, algorithms could be viewed as species. Each use of the same algorithms only differs in its starting condition (i.e., data provided by the experimenter and data extracted from the environment). Some general rules may emerge from studying the same algorithm, which may lead to replicable experimental results. In contrast, studying different algorithms could be viewed as studying the behavior of different species. Given their emphasis on the study of the behavior of individuals, behavior analysts should apply their expertise to this novel class of subjects (i.e., machines).

\section{Consistent with Behavior Analytic Terminology}

To allow communication between scientists working in different domains, a science of machine behavior analysis should remain terminologically consistent. For example, assume that we have a robot designed to respond to visual and auditory stimuli. When the robot perceives a human in its environment, it says "hello", and the human interacts with it. When this same robot perceives a rat in the environment (in the absence of a human), the robot says "hello", but this is never followed by a subsequent interaction. Eventually, the robot learns to say "hello" only when 
it perceives a human in its environment. If we applied consistent terminology to the previous example, we could describe the presence of a human in the environment as a discriminative stimulus for the robot engaging in the behavior of saying "hello", and the human interacting with the robot as a positive reinforcer for this same machine behavior.

The prior example shows one of the challenges of using consistent terminology. Even though we use the same terms (e.g., "discriminative stimulus", "extinction”), the internal learning mechanisms may differ significantly between living organisms and machines. By keeping the same terminology, it may seem that we are introducing a cognitive bias to our analyses by anthropomorphising machines. Given that we are not attributing any mentalistic attributes to the machines, but simply describing the impact of environmental variables on their behavior, the terminology remains an accurate description of what we are observing. This is why behavior analysts use the same terminology to describe learning across humans and other living organisms. Hence, the application of behavior analytic terminology circumvents this cognitive bias.

Another argument in favor of keeping terminology consistent is that others have already appropriated our terminology to describe similar procedures. For example, computer engineers and scientists use the expression "reinforcement learning" when describing a process wherein a machine is more likely to engage in responses that will lead to a preferred outcome (i.e., this preferred outcome is programmed). Nevertheless, whenever the use of terms could be misleading, we recommend preceding the behavior analytic term with "machine" as we have done with behavior (e.g., machine behavior, machine reinforcement, machine shaping). Adopting this approach in ambiguous contexts may prevent misleading our audience to what type of process we are referring to. In the end, we argue that the risks of misinterpretation are lesser than 
adopting different terminology, which could make the domain mutually unintelligible with others.

\section{Grounded in the Philosophical Assumptions of Behavior Analysis}

As argued since the beginning of this paper, we suggest that machine behavior analysis be considered as a fifth domain of behavior analysis. As such, sharing philosophical assumptions with the other domains of behavior analysis appears essential to the development of a coherent and systematic science. The philosophical assumptions of behavior analysis help us avoid the pitfalls of mentalism. Providing a detailed description of philosophical underpinnings of behavior analysis is beyond the scope of this paper, but we refer the reader to Moore (2008) for an introduction to the topic. Nonetheless, we will draw parallels with some of the philosophical assumptions that behavior analysts contact through their initial training: selectionism, determinism, empiricism, parsimony, and pragmatism (Behavior Analyst Certification Board [BACB], 2017).

Machine behavior analysis adheres to selectionism by relying on the external environment as an explanatory variable. The environment selects the behavior of the machine in the same manner as consequences select the behavior of living organisms. Computer code obviously mediates machine behavior, but this area of research is left to engineers. The main interest of the behavioral scientist is how the external environment affects machine behavior. As with the behavior of living organisms, the environment determines the behavior of machines (i.e., determinism). With sufficient experimentation and access to all initial conditions of a machine, scientists should be able to predict machine behavior. As with the study of living organisms and other complex sciences (e.g., weather prediction), the problem is that it may be difficult to consider all starting conditions and their subsequent effects on machine behavior, 
which limits its predictability in practice. Empiricism involves the assumption that the only way to study a phenomenon is through our contact with the environment (Marr, 2008). Therefore, machine behavior analysis relies on our senses, and not on thought experiments, to observe and manipulate the environment to produce meaningful changes. This adoption does not mean that thought experiments cannot be used as a starting point for novel ideas, but that the only way to develop a coherent and replicable science is through contact with the environment.

A science of machine behavior analysis adheres to parsimony. Parsimony justifies the selection of one theory, or concept, over another as follows: "Where we have no reason to do otherwise and where two theories account for the same facts, we should prefer the one which is briefer, which makes assumptions with which we can easily dispense, which refers to observables, and which has the greatest possible generality" (Epstein, 1984, p. 119). Machine behavior analysis should strive to develop a parsimonious science to explain machine behavior by minimizing assumptions. Parsimony may prevent the development of unnecessary concepts, such as consciousness, to explain machine behavior. For example, assume that a machine is learning to greet someone online to help them with a problem. We notice that, over time, the machine selects greetings in a manner that optimizes the time that the person spends online. A parsimonious explanation may be that the machine selects its greeting based on its prior experience in similar situations, which have been associated with interactions of longer durations. A non parsimonious explanation would be that the machine has developed selfawareness, which leads it to select an appropriate greeting. The latter concept is less parsimonious as it requires more assumptions (i.e., the existence of self-awareness) than the initial explanation that relies on the observable environment. 
Philosophers have developed many different versions of pragmatism since the late $19^{\text {th }}$ century, which can make it difficult to define (Bacon, 2012; Lattal \& Laipple, 2003). Despite being a key concept taught to future practitioners of our science (BACB, 2017), behavior analysts do not necessarily agree on what being pragmatic means (Barnes-Holmes, 2000; Leigland, 2003; Moore, 2016; Schoneberger, 2016). This debate centers around the place for reality and truth within pragmatism as applied to behavior analysis. Nevertheless, a basic premise of pragmatism is that "the true value of a statement is a function of how well the statement promotes effective action" (Moore, 2008, p. 400). A pragmatic science values the extent to which it can control nature or the environment. In applied behavior analysis, this pragmatism transpires through its emphasis on social significance of behavior and the magnitude of its change (Baer et al., 1968; Lattal \& Laipple, 2003). In our prior definition of the science, both the centered on machine behavior and relevance characteristics underlie this pragmatic perspective. A science of machine behavior analysis is relevant if it promotes effective action on the behavior of the user for which it was designed. By sharing philosophical assumptions with the other domains of behavior analysis, machine behavior analysis may produce results that are coherent and consistent with the sciences that focuses on living organisms.

\section{Some Arguments in Favor}

To summarize, machine behavior analysis is a science that examines how machines interact with and produce relevant changes in their external environment by relying on replicability, behavioral terminology, and the philosophical assumptions of behavior analysis (e.g., selectionism, determinism, parsimony) to study artificial behavior. Although this definition provides some boundaries for machine behavior analysis, a follow-up question is: Why should 
behavior analysts care about machine behavior? The next sections present arguments in support of encouraging more research on machines from a behavior analytic perspective.

\section{Machines Are Here to Stay and Just Keep Getting Better}

Machines are an increasing part of every domain of our daily lives. In 2015, there was approximately two connected devices per human on earth (Safaei et al., 2017). Safei et al. (2017) estimated that the number of connected devices would increase to nine per human on earth by 2030. Similarly, researchers expect that the amount of electronic waste (e.g., broken smartphones, obsolete computers) generated by humans will more than double between 2011 and 2030 (Shittu et al., 2020). Both prior estimates were produced prior to the COVID-19 pandemic, which has only accelerated the adoption of electronic devices in multiple domains of our lives (Vargo et al., 2021). That said, an increase in the number of machines alone may not justify the study of their behavior. As discussed previously, studying predictable machine behavior has limited relevance. The issue is that the number of machines has not only increased, but these machines are also getting much "smarter" and should keep doing so in the foreseeable future (Arif Wani et al., 2020; Hayhurst, 2019; Mammela, \& Anttonen, 2017). Put differently, both the amount of data taken as input and the algorithmic complexity of machines are increasing over time, which makes it more and more difficult to explain why they exhibit a specific response over another.

Some propositions for the future may push the limits of what machines may do and how they differ from humans. For example, researchers have recently developed a culturally competent robot that improves emotional well-being in older adults when compared to treatment as usual (Papadopoulos et al., 2021). Eventually, we can imagine robot carers that may reduce loneliness and support vulnerable populations in their independent living (Bardaro et al., 2021). 
For example, researchers and practitioners may develop and train robots to take care of both the physical and psychological needs of older adults (e.g., Costa et al., 2018; Niemelä \& Melkas, 2019; Papadopoulos et al., 2021). More ambitiously, the Alan Turing Institute (2020) proposed the AI Scientist Grand Challenge, which aims to develop a machine that could win a Nobel prize in science by 2050 . These more complex machines mean that engineers are not necessarily able to explain why a machine exhibits one behavior rather than another (or not at all) when provided with a specific input (von Eschenbach, 2021; Wadden, 2021). Given recent developments in artificial intelligence, the question is not if, but when machines will be able to have interactions that are indistinguishable from humans. Thus, behavior analysts should begin studying the behavior of machines now and develop tools to do so as they will become more complex (and unpredictable when a computer scientist looks at the code only) in the future.

\section{Machines Are Already Changing Human Behavior}

As one of the basic assumptions of behavior analysis is that the environment evokes, elicits or selects human behavior, we can easily argue that machines are already changing behavior. Amongst the most popular machines with which we interact daily, Amazon recommends products based on your prior purchases, Google autocompletes your search queries and provides results based on your history, Facebook relies on what you like to suggest news that should watch, and your Alexa smart speaker adapts its responses to your interaction with it. Therefore, machines can have a socially significant impact on our behavior for better (e.g., recommending a mental health provider to someone who displays behaviors associated with depression) or worse (e.g., recommending a news outlet that promotes bigotry or some unvalidated treatment). 
The applied and experimental sciences already study the interaction between humans and machines by focusing mainly on the behavior of the former (e.g., Critchfield \& Perone, 1990; Dallery et al., 2021; Higbee et al., 2016). This reality begs the question, why should we have a science of machine behavior analysis if we already study interactions between humans and machines? The response is that there are many situations in which we would want to isolate machine, rather than human, behavior. In these situations, we reverse the role of each variable in our inquiry. The machine behavior becomes the dependent variable whereas the human behavior is the independent variable. This approach contrasts with traditional studies with machines in behavior analysis wherein the dependent variable is the human behavior and the machine functions as the independent variable. For the same reasons that experimental analysts study behavior in the laboratory to better control for the effects of confounding variables, studying machine behavior on its own appears essential to contribute to our understanding of its interaction with its external environment.

\section{Engineers Do Not Have All the Answers}

Typically, we perceive machine behavior as being the concern of computer scientists or engineers. Engineers have a clear and central role in machine behavior: They are the ones who develop the hardware and code the software that the machines use to behave. However, we are reaching a point where computer scientists and engineers are unable to predict what behavior a machine will engage in following specific inputs, which is even leading to a crisis as to whether machines should be trusted with important decisions (von Eschenbach, 2021; Wadden, 2021; Wiens et al., 2019). Moreover, engineers are trained to change machine behavior through coding algorithms, but are not specialized in modifying the physical and social environment to alter 
behavior. Because machine behavior may also be modified by altering the environment (other than coding), we need a science beyond computer engineering to investigate machine behavior.

Behavior analysts are uniquely trained and positioned to address both the previous issues. First, researchers in behavior analysis have developed expertise and methodology in studying a subject that has a similar input (environment) and output (behavior). Similarly, behavioral scientists may look beyond the algorithms and coding (as we do with the brain and nervous system), and examine how the input provided to machines influences the output. Second, we are experts in how to modify behavior using the social and physical environment. As machines are being increasingly designed to interact with humans in a manner similar to other humans, behavior analysts may apply their knowledge and experience to modify machine behavior without the use of coding. This involvement may eventually lead to machines responding in a manner that is more beneficial to its users.

\section{Scientists Need a Non-Mentalistic Alternative to Explain Machine Behavior}

In his book Contingencies of Reinforcement, Skinner (1969) notably wrote "The real question is not whether machines think but whether men do" (p. 288). This phrase embodied the behavior analytic perspective involving the attribution of meta-physical properties to the human mind. In 1950, Alan M. Turing had anticipated a similar problem with machines when considering the question, “Can machines think?”. Similarly to Skinner, he found the question to be ambiguous and replaced it by the imitation game, which is now more commonly known as the Turing Test (Pinar Saygin et al., 2000). Unfortunately, this proposition by Turing (1950) did not end efforts at using a mentalistic approach to explain machine behavior. As examples, some researchers have begun to attribute personality traits to explain the types of behavior exhibited by machines (Qian et al., 2018; Lake et al., 2017). Furthermore, philosophers and scientists have 
continued to ponder if and when machines will show consciousness despite being rejected by Turing in 1950 (e,g., Gamez, 2008; Harnard, 2003; McCarthey, 1995; Signorelli, 2018; Wilks, 1984).

If behavior analysts fail to recognize the importance of machine behavior, the risk is that engineers will turn to mentalistic approaches in an attempt to explain and control machine behavior (see Bucher, 2017, for example). As noted previously, this reliance has already begun with studies on the personality of machines and on philosophical questions that focus on consciousness rather than on measurable and observable outputs. To prevent recourses to circular reasoning and asking questions that cannot be tested, we need a science whose locus of analysis is the social and physical environment of the machine. We argue that a science of machine behavior analysis would be well positioned to provide a non-mentalistic alternative to study the behavior of machines. This rejection of mentalism, and of meta-physical explanations, makes a science of machine behavior more practical and useful in producing changes that will eventually benefit humanity.

\section{A Science Requires an Organized Community}

One alternative to formalizing a science of machine behavior analysis is to incorporate it within an existing domain of behavior analysis, such as the experimental analysis of behavior or applied behavior analysis. One problem with this integration is that the focus of these domains is the behavior of living organisms. At this point, we have no reason to believe that all machines will behave in the same way as humans, or other living organisms, in the presence of specific environmental stimuli. Although the philosophy and methodologies of behavior analysis appear relevant to a science of machine behavior analysis, the processes and patterns uncovered for 
machines may differ considerably from cell-based organisms. As such, having a separate domain would allow for the eventual development of a knowledge base specific to machines.

The $20^{\text {th }}$ century has seen the development of many theories of knowledge to explain what constitutes a science such as Popper's falsification (1934/2002), Kuhn's paradigm shift (1962/2012), and Rorty's perspective on pragmatism (1979/2017). One commonality between these epistemologies is that a science develops within an organized community. To develop and to grow, sciences must be organized around a community who intersubjectively debate, discuss and agree on facts and ideas. In more behavior analytic terms, a science constitutes a verbal community that shares rules and contingencies acquired through contact with the environment (i.e., experiences and training). When the words, rules and contingencies between domains differ sufficiently, a new science typically emerges. Our position is that machine behavior differs enough from the behavior of living organisms to at least discuss the relevance of having its own domain.

To be clear, researchers have already conducted studies that meet most, if not all, the characteristics that we described previously. More than 20 years ago, Saksida et al. (1997) proposed using the principles of reinforcement to condition and shape robot behavior, albeit from an engineering standpoint. In the same year, Burgos (1997) discussed the training of artificial networks involving Pavlovian conditioning processes. In other words, some researchers are already contributing to machine behavior analysis, even though they may not refer to it as such. From philosophical and theoretical standpoints, behavioral scientists have previously speculated on important questions regarding machines such as whether we can make a machine "human" (Hutchinson, 2012; Rachlin, 2012) and whether having a nervous system is a necessary precondition for learning (Burgos, 2018). From an empirical standpoint, behavior analytic 
journals have published several studies simulating the behavior of machines and how they compare to the behavior of living organisms (e.g., Burgos, 2007; Lyddy et al., 2002; Ninness \& Ninness, 2020; Vernucio \& Debert, 2016). These examples are not exhaustive as the purpose of our paper was not to provide a systematic review of prior research in machine behavior analysis. Our proposal here is to organize and formalize this science within a community to accelerate and promote the development of this research domain.

\section{Some Future Directions}

Most exemplars of machine behavior analysis from the behavior analytic literature involve experimental work to simulate models of behavior. Behavior analysts may also study machines that already exist, or machines designed to solve problems of social significance. One potentially fertile area for future research is the study of machines designed to shape consumer behavior. Everyday, hundreds of millions of consumers use web-based applications such as Facebook, Netflix, Spotify, Amazon, TikTok, and Twitter. Because the code and algorithms of these apps are proprietary, we have limited knowledge on how these machines respond to human input. As behavioral shaping between machine and human is often a two-way interaction, studying how these machines respond to human behavior appears essential to improve their potential effects (Bucher, 2017).

Another area of research is health recommendation systems. Researchers are increasingly developing machines to support clinical decision-making (Wiens et al., 2019). One behavior analytic exemplar is a tool designed by Lanovaz et al (2020) to determine whether an AB graph shows a clear change. This web app may be used by mental health professionals to decide whether to continue or interrupt a behavioral intervention. The researchers who developed the app showed that their machine may make better decisions than humans, but a problem that 
remains is that we have no idea what type of decision errors this machine makes. To examine this question, we need to study the machine's behavior in isolation when given different inputs. Recommendation systems may even adapt their responding over time based on human responding (Derakhshan et al., 2019), underlining the relevance of the topic for behavior analytic research.

Machines may also be used to personalize teaching strategies to a learner's characteristics and responding (Luan \& Tai, 2021). The issue is that some of the decision-making algorithms are proprietary and other algorithms function as black boxes, making it nearly impossible for a human observer to predict its behavior. Before using these types of applications in practice or research, behavior analysts may conduct studies to examine how the machine responds to different patterns of input. Systematically studying machine behavior is one way to identify how these machines will interact with us and clients, which may ultimately lead to better decisions when selecting one technological alternative over another. Ethically, behavior analysts may also need to understand of how a learning application operates prior to the implementing it with their students.

Engineers are developing machines that are increasingly designed to behave and interact like humans. As noted earlier, some behavior analysts have even developed models that exhibit patterns similar to those observed in humans (e.g., Burgos, 2007; Lyddy et al., 2002; Ninness \& Ninness, 2020; Vernucio \& Debert, 2016). Two categories of machines that are often designed to behave like humans are chatbots and social robots. Thus, one fruitful area of research could be to study the verbal behavior of chatbots. How do chatbots respond to a human given different antecedents and consequences? Similarly, behavioral scientists may study the behavior of social robots who are designed to interact with, or even replace, humans in complex tasks. 
Manipulating the environment to examine how it reacts to changes is essential to ensure that these robots actually engage in behavior beneficial to humans. It should be noted here that the possibilities to study machine behavior are endless. The previous directions for future research are provided as examples as to how a science of machine behavior analysis could contribute to the advancement of behavior analysis.

\section{Conclusion}

Skinner (1969) argued that "man is a machine, but he is a very complex one" (p. 294). Our proposal for a science of machine behavior analysis remains consistent with this original conceptualization of man. At the time, machine behaviors were probably not complex enough to warrant their own domain. With the evolution of computing power and algorithms, we have already reached a point where an understanding of engineering is insufficient to explain and predict machine behavior. More importantly, we need a debate on the topic, regardless of whether behavior analysts agree or disagree with our position. Do we need a fifth domain? Do the proposed characteristics bound the science adequately? There is no doubt that behavioral researchers are already conducting studies and having academic discussions on the topic. The current article aims to encourage and contribute to this debate. Ultimately, a science of machine behavior analysis may help shape the behavior of machines to better meet the needs of humanity. 


\section{References}

Alan Turing Institute. (2020, February). AI scientist grand challenge.

https://www.turing.ac.uk/sites/default/files/2021-

02/summary_of_discussion_workshop_2020_ai_scientist_grand_challenge_clean.pdf

Arif Wani, M., Kantardzic, M., \& Sayed-Mouchaweh, M. (2020). Trends in deep learning applications. In Deep learning applications (pp. 1-7). Springer. https://doi.org/10.1007/978-981-15-1816-4_1

Bacon, M. B. (2012). Pragmatism: An introduction. Polity Press.

Baer, D. M., Wolf, M. M., \& Risley, T. R. (1968). Some current dimensions of applied behavior analysis. Journal of Applied Behavior Analysis, 1(1), 91-97. https://doi.org/10.1901/jaba.1968.1-91

Bardaro, G., Antonini, A., \& Motta, E. (2021). Robots for elderly care in the home: A landscape analysis and co-design toolkit. International Journal of Social Robotics. Advance online publication. https://doi.org/10.1007/s12369-021-00816-3

Barnes-Holmes, D. (2000). Behavioral pragmatism: No place for reality and truth. The Behavior Analyst, 23(2), 191-202. https://doi.org/10.1007/BF03392010

Behavior Analyst Certification Board. (2017). BCBA task list (5th ed.). Author. https://www.bacb.com/wp-content/uploads/2020/08/BCBA-task-list-5th-ed-211019.pdf

Bucher, T. (2017). The algorithmic imaginary: Exploring the ordinary affects of Facebook algorithms. Information, Communication \& Society, 20(1), 30-44. https://doi.org/10.1080/1369118X.2016.1154086

Burgos, J. E. (1997). Evolving artificial neural networks in Pavlovian environments. In J. W. Donahue \& V. Packard Dorsel (Eds.), Neural network models of cognition: 
Biobehavioral foundations (Vol. 121, pp. 58-79). North-Holland. https://doi.org/10.1016/S0166-4115(97)80090-8

Burgos, J. E. (2018). Is a nervous system necessary for learning?. Perspectives on Behavior Science, 41(2), 343-368. https://doi.org/10.1007/s40614-018-00179-7

Cooper, J. O., Heron, T. E., \& Heward, W. L. (2020). Applied behavior analysis (3rd ed.). Pearson.

Costa, A., Martinez-Martin, E., Cazorla, M., \& Julian, V. (2018). PHAROS-PHysical assistant RObot system. Sensors, 18(8), 2633. https://doi.org/10.3390/s18082633

Critchfield, T., \& Perone, M. (1990). Verbal self-reports of delayed matching to sample by humans. Journal of the Experimental Analysis of Behavior, 53(3), 321-344. https://doi.org/10.1901/jeab.1990.53-321

Dallery, J., Stinson, L., Bolívar, H., Modave, F., Salloum, R. G., Viramontes, T. M., \& Rohilla, P. (2021). mMotiv8: A smartphone-based contingency management intervention to promote smoking cessation. Journal of Applied Behavior Analysis, 54(1), 38-53. https://doi.org/ 10.1002/jaba.800

Derakhshan, B., Mahdiraji, A. R., Rabl, T., \& Markl, V. (2019). Continuous deployment of machine learning pipelines. In Proceedings of the 22nd International Conference on Extending Database Technology (pp. 397-408). https://openproceedings.org/2019/conf/edbt/EDBT19_paper_23.pdf

Epstein, R. (1984). The principle of parsimony and some applications in psychology. The Journal of Mind and Behavior, 5(2), 119-130.

Gamez, D. (2008). Progress in machine consciousness. Consciousness and Cognition, 17(3), 887-910. https://doi.org/10.1016/j.concog.2007.04.005 
Harnad, S. (2003). Can a machine be conscious? How?. Journal of Consciousness Studies, 10(45), 69-75.

Hayhurst, C. (2019). Getting smarter: The promise and potential of artificial intelligence in healthcare. Biomedical Instrumentation \& Technology, 53(2), 92-101. https://doi.org/10.2345/0899-8205-52.2.92

Higbee, T. S., Aporta, A. P., Resende, A., Nogueira, M., Goyos, C., \& Pollard, J. S. (2016). Interactive computer training to teach discrete-trial instruction to undergraduates and special educators in Brazil: A replication and extension. Journal of Applied Behavior Analysis, 49(4), 780-793. https://doi.org/10.1002/jaba.329

Hutchison, W. R. (2012). The central role for behavior analysis in modern robotics, and vice versa. The Behavior Analyst, 35(1), 29-35. https://doi.org/10.1007/BF03392262

Kunh, T. S. (2012). The structure of scientific revolutions. University of Chicago Press. (Original work published 1962)

Lake, B. M., Ullman, T. D., Tenenbaum, J. B., \& Gershman, S. J. (2017). Building machines that learn and think like people. Behavioral and Brain Sciences, 40, e253. https://doi.org/10.1017/S0140525X16001837

Lanovaz, M. J., Giannakakos, A. R., \& Destras, O. (2020). Machine learning to analyze singlecase data: A proof of concept. Perspectives on Behavior Science, 43(1), 21-38. https://doi.org/10.1007/s40614-020-00244-0

Lattal, K. A., \& Laipple, J. S. (2003). Pragmatism and behavior analysis. In K. A. Lattal \& P. N. Chase (Eds.), Behavior theory and philosophy (pp. 41-61). Springer.

Leigland, S. (2003). Is a new version of philosophical pragmatism necessary? A reply to BarnesHolmes. The Behavior Analyst, 26(2), 297-304. https://doi.org/ 10.1007/BF03392083 
Levitas, D. (2013). Always connected: How smartphones and social keep us engaged. International Data Corporation (IDC). https://www.nu.nl/files/IDCFacebook\%20Always\%20Connected\%20(1).pdf

Luan, H., \& Tsai, C. C. (2021). A review of using machine learning approaches for precision education. Educational Technology \& Society, 24(1), 250-266.

Lyddy, F., Barnes-Holmes, D., \& Hampson, P. J. (2001). A transfer of sequence function via equivalence in a connectionist network. The Psychological Record, 51(3), 409-428. https://doi.org/10.1007/BF03395406

Mammela, A., \& Anttonen, A. (2017). Why will computing power need particular attention in future wireless devices?. IEEE Circuits and Systems Magazine, 17(1), 12-26. https://doi.org/10.1109/MCAS.2016.2642679

Marr, J. (2003). Empiricism.In K. A. Lattal \& P. N. Chase (Eds.), Behavior theory and philosophy (pp. 63-81). Springer.

McCarthy, J. (1995). Making robots conscious of their mental states. In M. T. Cox \& M. Freed (Eds.), Representing mental states and mechanisms: Technical report SS-95-05 (pp. 8996). AAAI Press.

Moore, J. (2008). Conceptual foundations of radical behaviorism. Sloan Publishing.

Moore, J. (2016). Behavior analytic pragmatism. The Journal of Mind and Behavior, 37(3/4), 219-245.

Niemelä, M., \& Melkas, H. (2019). Robots as social and physical assistants in elderly care. In M. Toivonen \& E. Saari (Eds.), Human-centered digitalization and services (pp. 177-197). Springer. https://doi.org/ 0.1007/978-981-13-7725-9_10 
Ninness, C., \& Ninness, S. K. (2020). Emergent virtual analytics: Modeling contextual control of derived stimulus relations. Behavior and Social Issues, 29(1), 119-137. https://doi.org/10.1007/s42822-020-00032-0

Papadopoulos, C., Castro, N., Nigath, A., Davidson, R., Faulkes, N., Menicatti, R., .Khaliq, A. A., Recchiutu, C., Battistuzzi, L., Randhawa, G., Merton, L., Kanoria, S., Chong, N.-Y., Kamide, H., Hewson, D., \& Sgorbissa, A. (2021). The CARESSES randomised controlled trial: Exploring the health-related impact of culturally competent artificial intelligence embedded into socially assistive robots and tested in older adult care homes. International Journal of Social Robotics. Advance online publication. https://doi.org/10.1007/s12369-021-00781-X

Pinar Saygin, A.., Cicekli, I., \& Akman, V. (2000). Turing test: 50 years later. Minds and Machines, 10(4), 463-518. https://doi.org/10.1023/A:1011288000451

Popper, K. (2002). The logic of scientific discovery. Routledge. (Original work published 1934)

Qian, Q., Huang, M., Zhao, H., Xu, J., \& Zhu, X. (2018). Assigning personality/profile to a chatting machine for coherent conversation generation. In Proceedings of the twentyseventh international joint conference on artificial intelligence (pp. 4279-4285). Association of Computing Machinery.

Rachlin, H. (2012). Making IBM's computer, Watson, human. The Behavior Analyst, 35(1), 116. https://doi.org/10.1007/BF03392260

Rorty, R. (2017). Philosophy and the mirror of nature. Princenton University Press. (Original work published 1979) 
Rudin, C. (2019). Stop explaining black box machine learning models for high stakes decisions and use interpretable models instead. Nature Machine Intelligence, 1(5), 206-215. https://doi.org/10.1038/s42256-019-0048-x

Safaei, B., Monazzah, A. M. H., Bafroei, M. B., \& Ejlali, A. (2017). Reliability side-effects in Internet of Things application layer protocols. In 2017 2nd international conference on system reliability and safety (pp. 207-212). IEEE. https://doi.org/10.1109/ICSRS.2017.8272822

Saksida, L. M., Raymond, S. M., \& Touretzky, D. S. (1997). Shaping robot behavior using principles from instrumental conditioning. Robotics and Autonomous Systems, 22(3-4), 231-249. https://doi.org/10.1016/S0921-8890(97)00041-9

Schoneberger, T. (2016). Behavioral pragmatism: Making a place for reality and truth. The Behavior Analyst, 39(2), 219-242. https://doi.org/10.1007/s40614-016-0052-y

Sendak, M., Elish, M. C., Gao, M., Futoma, J., Ratliff, W., Nichols, M., Bedoya, A., Balu, S. \& O’Brien, C. (2020). "The human body is a black box" supporting clinical decisionmaking with deep learning. In Proceedings of the 2020 conference on fairness, accountability, and transparency (pp. 99-109). Association for Computing Machinery. https://doi.org/10.1145/3351095.3372827

Shittu, O. S., Williams, I. D., \& Shaw, P. J. (2021). Global E-waste management: Can WEEE make a difference? A review of e-waste trends, legislation, contemporary issues and future challenges. Waste Management, 120, 549-563. https://doi.org/10.1016/j.wasman.2020.10.016

Signorelli, C. M. (2018). Can computers become conscious and overcome humans?. Frontiers in Robotics and AI, 5, 121. https://doi.org/10.3389/frobt.2018.00121 
Skinner, B. F. (1958). Teaching machines. Science, 128(3330), 969-977.

Skinner, B. F. (1961). Cumulative record. Appleton-Century-Crofts.

Skinner, B. F. (1969). Contingencies of reinforcement: A theoretical analysis. AppletonCentury-Crofts.

Turing, A. M. (1950). Computing machinery and intelligence. Mind, 59(236), 433-460. https://doi.org/10.1093/mind/LIX.236.433

Vargo, D., Zhu, L., Benwell, B., \& Yan, Z. (2021). Digital technology use during COVID-19 pandemic: A rapid review. Human Behavior and Emerging Technologies, 3(1), 13-24. https://doi.org/10.1002/hbe2.242

Vernucio, R. R., \& Debert, P. (2016). Computational simulation of equivalence class formation using the go/no-go procedure with compound stimuli. The Psychological Record, 66(3), 439-440. https://doi.org/10.1007/s40732-016-0184-1.

von Eschenbach, W. J. (2021). Transparency and the black box problem: Why we do not trust AI. Philosophy \& Technology. Advance online publication. https://doi.org/10.1007/s13347-021-00477-0

Wadden, J. J. (2021). Defining the undefinable: The black box problem in healthcare artificial intelligence. Journal of Medical Ethics. Advance online publication. https://doi.org/10.1136/medethics-2021-107529

Wakefield, J. (2016, March 24). Microsoft chatbot is taught to swear on Twitter. BBC News. https://www.bbc.com/news/technology-35890188

Watson, G. W., \& Calhoun, E. C. (1960). Brief history of computers. School Science and Mathematics, 60(2), 87-94. https://doi.org/10.1111/j.1949-8594.1960.tb08329.x 
Watson, D. S., Krutzinna, J., Bruce, I. N., Griffiths, C. E., McInnes, I. B., Barnes, M. R., \& Floridi, L. (2019). Clinical applications of machine learning algorithms: beyond the black box. BMJ, 364. https://doi.org/10.1136/bmj.1886

Wiens, J., Saria, S., Sendak, M., Ghassemi, M., Liu, V. X., Doshi-Velez, F., Jung, K., Heller, K., Kale, D., Saeed, M., Ossorio, P. N., Thadaney-Israni, S., \& Goldenberg, A. (2019). Do no harm: A roadmap for responsible machine learning for health care. Nature Medicine, 25(9), 1337-1340.

Wilks, Y. (1984). Machines and consciousness. In Hookwya, C., Minds, machines and evolution: Philosophical studies (pp. 105-128). Cambridge University Press. 


\section{Figure 1}

Diagram of a Simplified Machine

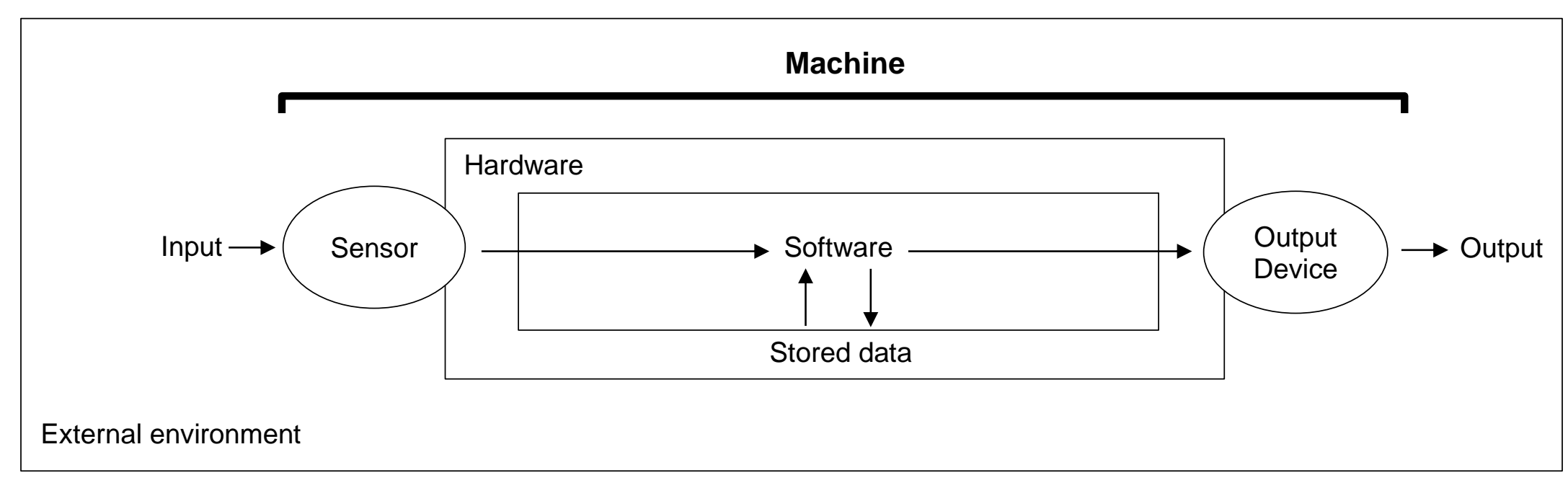

